\section{$\underset{\& \text { migrations }}{\text { hommes }}$}

\section{Hommes \& migrations}

Revue française de référence sur les dynamiques

migratoires

$1304 \mid 2013$

Frontières

\title{
Retour sur la Marche pour l'égalité et contre le racisme
}

\section{Abdellali Hajjat}

\section{(2) OpenEdition}

\section{Journals}

Édition électronique

URL : http://journals.openedition.org/hommesmigrations/2677

DOI : $10.4000 /$ hommesmigrations. 2677

ISSN : 2262-3353

\section{Éditeur}

Musée national de l'histoire de l'immigration

\section{Édition imprimée}

Date de publication : 1 octobre 2013

Pagination : 151-155

ISBN : 978-2-919040-24-7

ISSN : $1142-852 X$

\section{Référence électronique}

Abdellali Hajjat, «Retour sur la Marche pour l'égalité et contre le racisme», Hommes \& migrations [En ligne], 1304 | 2013, mis en ligne le 18 mars 2014, consulté le 22 septembre 2020. URL : http:// journals.openedition.org/hommesmigrations/2677; DOI : https://doi.org/10.4000/ hommesmigrations.2677 


\section{MÉMOIRES}

\section{RETOUR SUR LA MARCHE POUR L'ÉGALITÉ ET CONTRE LE RACISME}

ABDELLALI HAJJAT, enseignant-chercheur en sociologie, université de Paris-Ouest-Nanterre.

Le trentième anniversaire de la Marche pour l'égalité et contre le racisme (15 octobre-3 décembre 1983) est l'occasion de multiples commémorations publiques d'un événement fondateur de l'histoire de l'immigration en France : film réalisé par Nabil Ben Yadir, documentaires télévisés, reportages radiophoniques, numéros de revue et colloques, ouvrages de témoignages, réunions publiques, expositions, etc. La "Marche" est devenue un objet de mémoire où se mêlent et s'affrontent les discours de multiples protagonistes: acteurs de l'époque longtemps restés silencieux (en particulier Toumi Djaïdja, initiateur de la Marche), militants immigrés, journalistes, membres du gouvernement, artistes, etc. Grâce à cette commémoration à grande échelle, le grand public prend connaissance d'un événement dont le souvenir était jusque-là cantonné à des cercles confidentiels. En ce sens, le trentième anniversaire semble marqué par un changement d'échelle et une pluralité des points de vue. La profusion des discours publics sur la Marche n'est pas sans véhiculer des contre-vérités historiques et des reconstructions de l'événement révélatrices des enjeux politiques de notre époque'. Ainsi, la politisation de la question musulmane et la montée de l'islamophobie favorisent la construction, par certains acteurs associatifs, médiatiques, voire académiques, de l'opposition entre les "Beurs laïques", censés être représentés par les marcheurs, et les "musulmans inassimilables" des banlieues. Dans un autre registre, les marcheurs et le mouvement "beur" sont souvent accusés de "naïveté" et d'avoir été "facilement récupérés" par l'association SOS Racisme, satellite du Parti socialiste.

Au-delà du mythe positif ou négatif de la Marche, tout le monde s'accorde à dire qu'elle symbolise l'apparition des enfants d'immigrés postcoloniaux dans l'espace public français. Auparavant, l'immigration maghrébine était généralement considérée comme un phénomène provisoire et représentée dans l'opinion publique par la figure du travailleur immigré, célibataire et sans enfants ${ }^{2}$, occultant ainsi notamment la présence féminine dans l'immigration³. À partir du début des années 1980, les enfants d'immigrés postcoloniaux font la Une de l'actualité, notamment lors des rébellions urbaines de la banlieue lyonnaise et de la grève de la faim des jeunes de SOS Avenir Minguettes 4 . 
Pour la première fois dans l'histoire de France, cette catégorie de la population va faire l'objet d'un discours médiatique et politique au niveau national, et la manifestation finale à Paris, qui rassemble environ cent mille personnes, produit un immense espoir et un unanimisme antiraciste dans l'opinion publique. Si le traitement médiatique de l'événement est bien connu's, cet article propose de compléter les écrits existants ${ }^{6}$ en revenant brièvement sur les conditions de possibilité de cette action collective. Notre perspective sociohistorique cherche à inscrire la Marche dans une histoire longue, au croisement de l'histoire des classes et des banlieues populaires (en particulier celle de la ZUP des Minguettes à Vénissieux), de l'histoire de l'immigration postcoloniale, de l'histoire des mobilisations dans le sillage des "années 19687" et de l'histoire politique française.

\section{Des Minguettes à l'Élysée}

Les origines de la Marche doivent être situées dans les événements des Minguettes de l'été 1981 et de mars-avril 1983. Cette ZUP (zone à urbaniser en priorité) emblématique de la banlieue lyonnaise constitue un véritable laboratoire où les relations entre la classe ouvrière stabilisée (ouvriers professionnels et employés) et la classe ouvrière précarisée (ouvriers spécialisés et jeunes chômeurs) se détériorent progressivement durant les années 1970. Dans un contexte de crise économique et de montée du chômage des jeunes, l'exacerbation des tensions sociales est le résultat d'un processus social complexe lié aux modalités de peuplement des immeubles, au phénomène de mobilité résidentielle, à la politique discriminatoire d'attribution des logements sociaux, à la logique de ségrégation sociale et ethnique et à la crise d'encadrement de la jeunesse ouvrière.

Ces transformations de l'espace social des Minguettes favorisent une "lutte des classes interne" à la classe ouvrière, c'est-à-dire des conflits entre la fraction la plus aisée des classes populaires et les bandes de jeunes enfants d'ouvriers spécialisés, qui constituent le vivier des rebelles de 1981 et 1983.

Durant l'été 1981, des centaines de jeunes défient la police et participent à une rébellion urbaine devenue le symbole de la "crise des banlieues". Le 21 mars, une deuxième rébellion rassemble des centaines d'habitants du quartier Monmousseau, jeunes et mères de famille, pour dénoncer les déviances policières violentes. Après le retrait des forces de police, les rebelles se transforment en manifestants en organisant une marche et un sit-in devant le commissariat et la mairie de Vénissieux dirigée par le maire communiste Marcel Houël.

Il s'ensuit l'organisation d'une grève de la faim, puis la création de l'association SOS Avenir Minguettes par les jeunes du quartier, qui parviennent, par l'entremise du prêtre Christian Delorme, à nouer un dialogue avec le gouvernement de Pierre Mauroy. Mais le rapport de force avec la mairie communiste, hostile aux grévistes, et avec les forces de l'ordre, qui ne reconnaissent pas les violences policières, conduit à une impasse au niveau local.

Les tensions sociales continuent aux Minguettes et atteignent leur paroxysme lorsque, le 19 juin,

5. Alain Battegay, Ahmed Boubeker, Les Images publiques de l'immigration. Médias, actualité, immigration dans la France des années 1980, Paris, L'Harmattan, 1993. 6. Notamment Adil Jazouli, L’Action collective des jeunes Maghrébins de France, Paris, L'Harmattan, 1986 ; Nicolas Beau, Ahmed Boubeker, Chroniques..., op. cit. ; Saïd Bouamama, Dix ans de marche des Beurs. Chronique d'un mouvement avorté, Paris, Desclée de Brouwer, 1994 ; Ahmed Boubeker, Les Mondes de l'ethnicité. La communauté d'expérience des héritiers de l'immigration maghrébine, Paris, Balland, 2003 ; Stéphane Beaud, Olivier Masclet, “Des 'marcheurs' de 1983 aux 'émeutiers' de 2005 . Deux générations sociales d'enfants d'immigrés", in Annales. Histoire, sciences sociales, $n^{\circ} 4$, 2006 , pp. 809-843; Mogniss H. Abdallah, Rengainez, on arrive !, Paris, Libertalia, 2012. 
le président de l'association, Toumi Djaïdja, se fait tirer dessus par un policier. L'idée d'une marche devient évidente pour SOS Avenir Minguettes et les militants de la Cimade de Lyon, pour sortir de l'impasse locale.

Si la Marche parvient à mobiliser plusieurs milliers de personnes à travers toute la France, c'est d'abord en raison du soutien des Églises catholique et protestante et du mouvement pro-immigrés (Fasti, Mrap, etc.), qui assurent l'accueil des marcheurs au fil des étapes. Du côté des associations de jeunes immigrés, c'est le scepticisme qui règne, voire la suspicion envers une supposée "manipulation des curés", mais les militants immigrés finissent par y participer en créant des "Forums justice" et des "Collectifs jeunes".

La Marche bénéficie aussi de l'appui de quelques journalistes (Antenne 2, Le Monde, Libération, etc.) qui assurent une couverture médiatique très favorable dès le début. Mais c'est lors de l'assassinat de Habib Grimzi par des légionnaires dans le train Bordeaux-Vintimille (14 novembre) que les grands médias s'intéressent vraiment à la mobilisation.

Cette attention médiatique provoque l'arrivée de personnalités nationales (ministres, dirigeants syndicaux, etc.), mais le gouvernement conserve une posture prudente à l'égard des marcheurs : il ne souhaite pas soutenir ouvertement une mobilisation qui pourrait mal tourner, mais il maintient officieusement des relations avec le groupe des marcheurs. Cette prudence socialiste se maintient jusqu'à ce que, à la fin de la manifestation parisienne, le président de la République accepte de recevoir une délégation de marcheurs. À l'issue de la discussion, les marcheurs annoncent que le chef de l'État accepte l'idée d'une carte de séjour de dix ans, instaurée en juillet 1984 et assurant une meilleure stabilité pour les étrangers (hors Communauté économique européenne).

\section{Les ambiguïtés de l'unanimisme antiraciste}

Malgré le succès quantitatif de la mobilisation et la satisfaction d'une revendication centrale du mouvement pro-immigrés, l'apothéose parisienne est marquée par des ambiguïtés qui se révèlent les années suivantes. Tout d'abord, la volonté de rassembler largement et de séduire

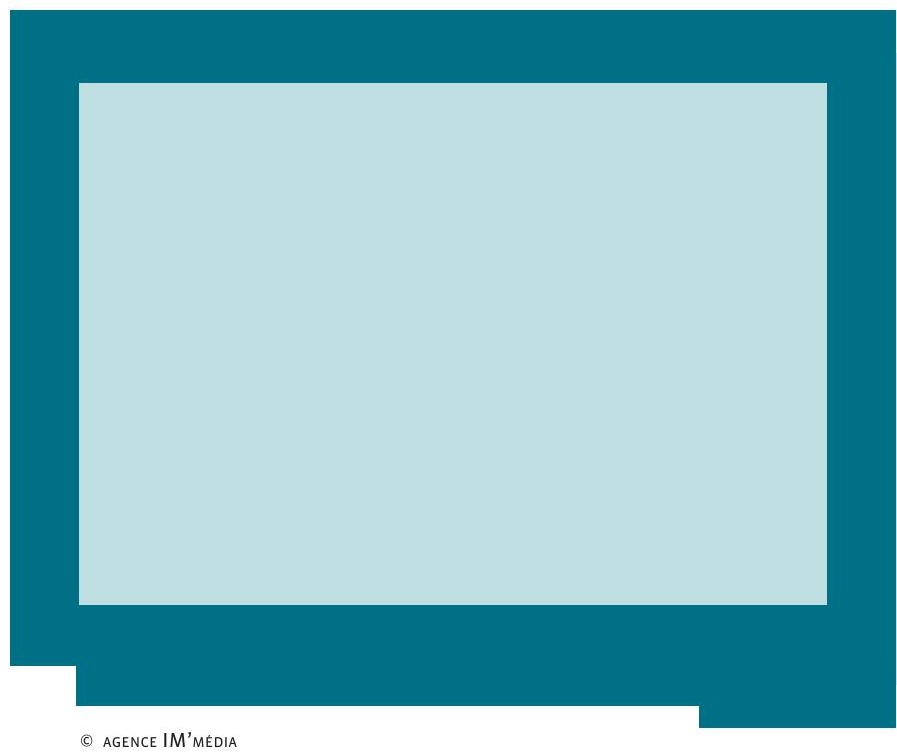

les médias conduit les marcheurs à produire un discours très général (droit à la vie) qui marginalise les enjeux qui étaient au fondement de SOS Avenir Minguettes : égalité de traitement par la police et la justice, droit au travail et au logement. Ainsi, les inégalités raciales et économiques sont globalement occultées dans les discours dominants, qui privilégient la question de la "différence" culturelle.

La "culturalisation" du débat public est la source d'une autre ambiguïté:la popularisation du terme 


\section{MÉMOIRES}

"beur". À l'origine, " Beur » signifie " Arabe » en verlan et le mot est utilisé par les enfants d'immigrés maghrébins de la région parisienne pour s'auto-désigner. Mais le terme échappe rapidement à ses inventeurs et devient une forme d'assignation identitaire. Les "Beurs" ne sont wplus des Arabes : ils ne sont ni des Français à part entière ni tout à fait des immigrés, et les "bons Beurs" se distinguent des "mauvais travailleurs immigrés". Le terme "beur" scelle la séparation symbolique d’avec la génération des parents immigrés. Au moment même où les "jeunes immigrés" font leur entrée symbolique dans l'espace public, les travailleurs immigrés sont disqualifiés symboliquement lors des grèves de l'automobile (Citroën-Aulnay et Talbot-Poissy). Ce basculement est fondamental dans l'histoire de l'immigration parce qu'il correspond à la construction de l'opposition

Force est de constater que les classes dominantes

françaises, malgré leur hétérogénéité et leurs divisions, ont répondu défavorablement à la quête d'égalité et de justice. L'appel de la Marche n'a pas été entendu. entre les "Beurs laïques assimilables" et les "immigrés musulmans inassimilables". Ainsi, l'année 1983 correspond aux débuts de la nouvelle construction du "problème musulman" articulée avec celle de "I'immigration". L'immense espoir provoqué par le surprenant succès de la manifestation finale de la Marche est vite remplacé par une exacerbation des tensions sociales dans les banlieues et la désillusion des marcheurs et des jeunes de SOS Avenir Minguettes. Certains continuent à se mobiliser dans le secteur associatif et/ou trouvent un emploi, d'autres sombrent dans la délinquance et/ou meurent prématurément, tandis que Djaïdja est l'objet d'un harcèlement policier et judiciaire débouchant sur son incarcération en 1984 (il est gracié par Mitterrand

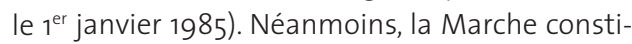
tue aussi un extraordinaire vecteur de sociali- sation politique pour toute une génération de militants des quartiers populaires et favorise la naissance de centaines d'associations de quartier et/ou de l'immigration et devient une référence historique pour tous les mouvements ultérieurs.

\section{Persistance des problèmes et actualité du combat}

Trente ans après la Marche pour l'égalité et contre le racisme, les aspirations exprimées par l'association SOS Avenir Minguettes et les associations de "jeunes issus de l'immigration" sont toujours d'actualité : l'égalité de traitement par la police et la justice, le droit au travail, au logement, à la citoyenneté et au séjour sont au cœur des nombreuses actions collectives qui se sont succédé après 1983. La quête d'égalité réelle et de justice sociale prend de multiples formes, qu'elles soient violentes ou non violentes, comme on a pu l'observer lors des rébellions urbaines de 2005. Celles-ci sont révélatrices de l'accroissement des tensions entre les classes sociales en France, conséquence d'une dégradation des conditions d'existence des classes populaires. Il est indéniable que la situation économique et sociale des quartiers d'habitat social s'est lourdement aggravée avec la crise du capitalisme industriel et financier, l'accroissement des inégalités, l'avènement du "précariat", l'affaiblissement de l'État social, le développement de l'économie de la drogue, etc.

Force est de constater que les classes dominantes françaises, malgré leur hétérogénéité et leurs divisions, ont répondu défavorablement à la quête d'égalité et de justice. L'appel de la Marche n'a pas été entendu. Au lieu d'ouvrir des brèches dans les structures de domination économique, politique, nationale et culturelle, l'exigence d'égalité portée par les enfants d'immigrés postcoloniaux a provoqué une crainte et un ressentiment 
de la part des "élites". Les désillusions des marcheurs s'expliquent par un processus bien connu de la sociologie des relations entre établis et marginaux: les tensions entre les groupes dominants et les groupes stigmatisés ne peuvent que s'accroître quand la quête d'égalité suppose la fin des privilèges, qu'ils soient économiques, raciaux ou de genre. Tant que les immigrés restent des ouvriers spécialisés corvéables à merci et que leurs enfants restent à leur place de bons "Beurs", la tension reste à un niveau relativement bas. Mais ce niveau monte dès que les membres d'un groupe marginal aspirent à s'élever socialement et politiquement.

En contexte migratoire, le refus de l'égalité se traduit par la remise en cause de la légitimité "présentielle" de l'immigration postcoloniale (elle n'est pas "chez elle"). Initiée dès les années 1960 pour endiguer l'immigration algérienne, la politique de "maîtrise des flux migratoires" s'est élargie à d'autres nationalités et s'est poursuivie jusqu'à violer les droits humains les plus élémentaires des étrangers non communautaires : droit à la vie de famille, au travail, à la santé, etc. La succession des lois sur l'immigration et la nationalité, avec son lot de durcissements des conditions de séjour et d'expulsions massives, n'est pas une réponse "démocratique" et "responsable" à la montée d'une "xénophobie populaire" et du Front national : elle révèle la croyance persistante des "élites", de droite comme de "gauche", en l'existence d'un "problème immigré". C'est ainsi que les frontières de la nation française se sont peu à peu refermées aux étrangers non communautaires, au point que le nombre d'expulsions atteint de nos jours des sommets inégalés (environ 30 ooo par an), que l'attribution de titres de séjour se fait au compte-gouttes et que le nombre de naturalisations a brutalement chuté au début des années 2010, pour la première fois depuis le régime de Vichy. Si les étrangers sont les premières cibles de cette "délégitimation présentielle", les enfants d'étrangers nés en France de parents étrangers, qui deviennent automatiquement français à leur majorité grâce au principe du droit du sol, sont aussi l'objet d'une attention particulière.

Dès le retour de la droite au pouvoir en 1986, le gouvernement de Jacques Chirac propose d'introduire le principe de "manifestation de volonté" aux enfants bénéficiaires du droit du sol, remettant en cause leur loyauté vis-à-vis de la République française. Bien qu'ils soient nés et élevés en France, ils ne sont pas considérés comme des Français à part entière et doivent montrer leur "volonté" d'être français.

Cette proposition provoque un tollé général dans l'opinion publique, ce qui amène le gouvernement à créer la Commission sur la nationalité, présidée par Marceau Long, dont les conclusions peuvent se résumer ainsi : l'immigration pose problème, mais l'intégration est en marche. L'idée dominante est qu'il faut "croire" dans la "capacité d'intégration" des institutions françaises, en particulier l'école publique, et que le processus d'"extinction" culturelle et religieuse est inexorable.

Les classes dominantes se sont donc senties "trahies" lorsque des enfants d'immigrés postcoloniaux, en particulier les jeunes filles, se sont mis à pratiquer la religion musulmane, à porter le hijab et à fréquenter la mosquée. De leur point de vue, la religiosité musulmane remet en cause la conception dominante de la nation française, fondée sur le principe de l'homogénéité nationale. C'est à partir de ce principe qu'il faut comprendre la dernière proposition de I'UMP de remettre en cause le droit du sol pour les enfants de sans-papiers ${ }^{7}$ : ceux-ci ne seraient pas véritablement "intégrables". 